Original article

\title{
Health-related quality of life among COVID-19 individuals: A cross-sectional study in Tamil Nadu, India
}

\author{
Suganya Barani ${ }^{\mathrm{a}}$, Tarun Bhatnagar ${ }^{\mathrm{b}}$, Meenakumari Natarajan ${ }^{\mathrm{b}}$, Kumari Gayathri ${ }^{\mathrm{b}}$, \\ Harshal Bhimrao Sonekar $^{\mathrm{b}}$, Akhil Sasidharan ${ }^{\mathrm{b}}$, T.S. Selvavinayagam ${ }^{\mathrm{a}}$, \\ Bhavani Shankara Bagepally ${ }^{\text {b,* }}$
}

${ }^{a}$ Directorate of Public Health and Preventive Medicine, Tamil Nadu, India

${ }^{\mathrm{b}}$ ICMR-National Institute of Epidemiology, Chennai, India

\section{A R T I C L E I N F O}

\section{Keywords:}

Quality of life

HRQOL

COVID-19

Cross-sectional

\begin{abstract}
A B S T R A C T
Introduction: The EQ-5D-5L questionnaire is a tool that is a very generic and preference-based instrument to describe the health-related quality of life. We have generated the stratified index utility value for the Tamil Nadu population and compared the utility values based on socio-demographic and clinical characteristics.

Methods: We conducted a community-based analytical cross-sectional study using telephonic interviews from November 2020 till December 2020 among individuals aged 18 years and above who were infected by the coronavirus confirmed with an RT-PCR within 30 days in Tamil Nadu State. EQ-5D-5L profile, sociodemographic and clinical characteristics of the study participants were collected and analysed.

Results: We interviewed 372 participants, with 57.5\% were males, and their mean age was $44.5 \pm 15.3$ years. About $40 \%$ of participants reported as having comorbidities, such as diabetes (19.4\%), hypertension (12.4\%), heart disease (2.4\%), kidney disease (0.8\%) and others. The mean EQ-5D utility score was $0.925 \pm 0.150$, and the mean EQ-VAS was $90.68 \pm 11.81$. Overall, men had a higher utility value $(0.938 \pm 0.130)$ than women, $(0.907$ \pm 0.170 ). Individuals with comorbidities, requiring longer hospitalisation were having lower utility scores than their counterparts.

Conclusion: We report the EQ-5D-5L utility values for the COVID-19 illness. These values would help to estimate quality-adjusted life years in health economic evaluation studies.
\end{abstract}

\section{Introduction}

The COVID-19 pandemic has become a crisis of historic proportions, posing major challenges to global health as well as stalled economic progress. ${ }^{1}$ There were 175 million confirmed cases of severe acute respiratory syndrome coronavirus 2 (SARS-CoV-2) until June 13, 2021, and it has caused 3.8 million deaths worldwide. ${ }^{2}$ In India, Tamil Nadu is one of the states with a higher caseload, with 149,927 active cases and 29, 547 deaths until June 13, 2021. Since March 2020, 2,174,247 individuals have recovered from COVID-19 in Tamil Nadu. ${ }^{3}$ The imposition of movement restrictions to halt the virus spread has disproportionately impacted the population. Thus, Covid-19 affected health, altered psychosocial behaviour, lifestyle changes, livelihood, and quality of life ${ }^{4,5}$ Health care interventions to combat the pandemic incurs costs from both public payers (governments) as well as individual payers (through out-of-pocket expenditure). Identification of cases, isolation, quarantine, contact tracing, and community interventions accrue considerable costs. To evaluate the effectiveness of all such interventions, universally accepted and the comparable outcome such as a standard measure of the quality of health status is essential to describe the health of the population affected by COVID-19 and would be of immense value.

Health-related quality of life (HRQOL) refers to the impact of disease and treatment on patients' function and overall life satisfaction. ${ }^{6}$ The EQ-5D-5L questionnaire is a generic and preference-based tool to describe \& quantify HRQOL based on the patient's perspective of their health. It describes the HRQOL status by five dimensions with five levels each and a visual analogue scale. Based on the population under study

\footnotetext{
* Corresponding author. Scientist-D, ICMR-National Institute of Epidemiology,Co-Ordinator, Health Technology Assessment Resource Centre ICMR-NIE, R-127, Tamil Nadu Housing Board, Phase I and II, Ayapakkam, Chennai, 600077, India.

E-mail address: bagepally.bs@gov.in (B.S. Bagepally).
} 
and value sets, an index value (utility value) is valuated using standard valuation technology. ${ }^{7}$ Such a health condition-specific (for COVID) utility score could be used to calculate the quality-adjusted life years (QALY) of health outcomes of any tested health interventions/technologies. This allows comparing the cost-effectiveness of the different interventions for COVID-19/health conditions. Thus aiding in generating economic literature for evidence-based based policy decision making. ${ }^{8}$

So far, there have been no studies generating utility scores for COVID-19 from India. In particular, COVID-19 and its impact on the quality of life have not been explored in Tamil Nadu. So in this study we estimated the EuroQol utility index value for COVID-19 recovered individuals at 30 days subsequent to confirmation of COVID-19 in Tamil Nadu. Further, we estimated the influence of demographic, socioeconomic, and clinical factors on EuroQol utility index value in the recovered individuals at 30 days subsequent to confirmation of COVID-19 in Tamil Nadu. This will help inform the policymakers about differences in the value of health status as perceived by the groups in the Tamil Nadu population and provide the utility scores of COVID-19 for conducting economic evaluation studies.

\section{Methods}

The STROBE (Strengthening the Reporting of Observational Studies in Epidemiology) guidelines were followed during the preparation of this manuscript. ${ }^{9}$

Study Design and study participants: A community-based analytical cross-sectional study was conducted from November 2020 till December 2020 among individuals aged 18 years and above who were infected by the coronavirus confirmed with an RT-PCR within 30 days in Tamil Nadu State.

Sampling: We sampled the population using a simple random sampling method from the COVID-19 line-list maintained by the Department of Public Health, Tamil Nadu. Since the Indian population had no utility index as a reference, we calculated the sample size using the ordinary least square model ${ }^{10}$ with a design effect of $0.75,0.05$ (MID) as the half-width of the $95 \%$ PI of the estimated utility value, and non-response of $10 \%$. We selected 420 individuals across Tamil Nadu. The institutional human ethics committee (NIE/IHEC/202010-02) of the National Institute of Epidemiology approved this study, and all subjects provided informed consent.

Data Collection: We collected data by telephonic interview using a structured questionnaire in both English and Tamil language. An Open Data Kit (ODK) software was used for data entry. Socio-demographic characteristics collected in the interview included age, gender, area of residence, educational attainment, employment status, and marital status. Anthropometric factors such as height and weight, details on hospitalisation, treatment, and the number of days of respective stay were also collected.

Measurement of EQ- 5D-5L: We collected HRQOL using the EQ-5D5L tool. The EQ-5D is a standardized, generic HRQOL instrument. The first part divides health into five dimensions: mobility, self-care, usual activities, pain/discomfort, and anxiety/depression. Each dimension is divided into five levels; no problem (level 1), slight problem (level 2), moderate problem (level 3), severe problem (level 4), and extreme problem (level 5). ${ }^{11}$ The second part of the EQ-5D is the EQ visual Analogue scale (EQ-VAS). ${ }^{12}$ It records the respondent's self-reported health on a vertical visual analogue scale (VAS), where $0-100$ represent the worst and best imaginable health states, respectively. ${ }^{13}$ In the telephonic interview, we requested the participants to imagine a scale of value zero to one-hundred and to describe their health state with a number between 0 and 100 to get the VAS value. This study used the Thailand value set and scoring algorithm to calculate utility scores by crosswalk methodology for each patient ${ }^{14}$ since an Indian scoring algorithm is not yet available for the EQ-5D-5L. A utility index of one denotes perfect health, and zero represents death.
We recorded participants as non-response if they did not have a telephone number or were hospitalized during the call/interview and those who were not available at the time of the call. If a participant is not picking the call for the first time, three subsequent attempts were made with an interval of $20 \mathrm{~min}$. Failure to respond after the final attempt lead to declaring the participant as non-response. If the participants are unable to undergo the telephonic interview at the time of call, the telephonic interview was rescheduled based on a mutual consensus. If the participant was not feeling well, he/she could nominate a family member as a respondent to answer and continue.

We used the EQ-5D-5L crosswalk index value calculator from the Euro QoL website to obtain the EQ-5D utility value. We used the WHO BMI criteria for classifying into as underweight, normal, overweight, and obese. We considered town panchayat under the rural area of residence even though town panchayat is the body of government for areas in transition from rural to urban, and Tamil Nadu is the first state to introduce such a classification.

Data analysis: Data analysis was performed using Statistical Package for the Social Sciences (SPSS version-23). Descriptive summary statistics were estimated for socio-demographic variables, the EQ-5D-5L dimensions, utility scores, EQ-VAS scores. We described individual demographic characteristics using frequencies and percentages and utility values by mean and standard deviation. EQ-5D-5L utility scores and EQVAS were tested for Normality (Shapiro-Wilk test), and differences in relationships of all factors and the scores of EQ-5D were analysed using the non-parametric Mann Whitney $U$ test (two groups) and KruskalWallis one way analysis of variance (multiple groups). We did Fischer's exact test to explore the statistical significance of the difference between the groups in the percentage of reported problems with EQ5D Utility scores and EQ-VAS. We performed a multivariate logistic regression model by using five dimensions as dependent variables after dichotomization $(0=$ no problem, $1=$ problem $)$. We set a statistical significance of 0.05 (two-sided test) for all calculations. A p-value of less than 0.05 was considered statistically significant.

\section{Results}

Characteristics of the Study Participants: We interviewed 372 individuals, and their mean age was $44.5 \pm 15.3$ years. The majority of participants $(81.1 \%)$ were aged above 30 years and married $(82.5 \%)$. More than half of the participants were males (57.5\%). Nearly half of the participants had received education up to the 12 th class (49.5\%). More than half of our study participants were overweight and obese combined (55\%). Around one-fifth of study participants were diabetic (19.4\%), and more than one-tenth of participants were hypertensive $(12.4 \%)$. Nearly $40 \%$ of the participants reported having comorbidity (Table 1 ). More than three quarter (83\%) of participants reported being in-home quarantine at one point in time. About $41 \%$ of the participant's used government hospitals, and nearly one-third of participants (33\%) used private hospitals with a median number of seven days of stay. The participants reported staying in a private hospital for up to 40 days when in comparison, the maximum number of days of stay at a government facility was under 30 days.

EQ-5D-5L results: EQ-5D-5L utility score and EQ-VAS score were not normally distributed at each time point, as assessed by ShapiroWilk's test ( $\mathrm{p}>0.05)$. The mean EQ-5D index score (utility score) for all individuals was $0.925 \pm 0.150$, and the mean EQ-VAS was $90.68 \pm$ 11.81 (Table 1). Overall there was no significant difference in utility score and EQ-VAS score. However, individuals with comorbidities reported low EQ-5D index scores; Kidney diseases $(\mathrm{p}<0.05)$ showed a significant association, with lower EQ-5D scores $0.77 \pm 0.20$ (Table 1 ). Most study participants reported pain/discomfort (16\%), followed by anxiety/depression (12.4\%). Individuals aged 60 years or above reported problems consistently in all domains such as self-care $(6.1 \%)$, usual activities (10.6\%), pain/discomfort (21.2\%), and anxiety/ depression $(12.1 \%)$. Individuals engaged in domestic work reported 
Table 1

Characteristics of respondents and EQ-5D index and visual analogue (VAS) scores.

\begin{tabular}{|c|c|c|c|c|c|}
\hline \multirow[t]{2}{*}{ Characteristics } & \multirow[t]{2}{*}{ N (\%) } & \multicolumn{2}{|c|}{ EQ-5D-5L Index } & \multicolumn{2}{|c|}{ EQ-5D-5L VAS } \\
\hline & & $\begin{array}{l}\text { Mean } \\
\text { (SD) }\end{array}$ & $\begin{array}{l}\mathrm{P} \\
\text { value }\end{array}$ & Mean (SD) & $\begin{array}{l}P \\
\text { value }\end{array}$ \\
\hline Total & $\begin{array}{l}372 \\
(100)\end{array}$ & $\begin{array}{l}0.93 \\
(0.15)\end{array}$ & & $\begin{array}{l}90.68 \\
(11.81)\end{array}$ & \\
\hline \multicolumn{6}{|l|}{ Gender } \\
\hline Male & $\begin{array}{l}214 \\
(57.5)\end{array}$ & $\begin{array}{l}0.94 \\
(0.13)\end{array}$ & $0.175^{\mathrm{a}}$ & $\begin{array}{l}91.57 \\
(11.11)\end{array}$ & $0.265^{\mathrm{a}}$ \\
\hline Female & $\begin{array}{l}155 \\
(41.7)\end{array}$ & $\begin{array}{l}0.91 \\
(0.17)\end{array}$ & & $\begin{array}{l}89.38 \\
(12.7)\end{array}$ & \\
\hline Other & $3(0.8)$ & $\begin{array}{l}1.00 \\
(0.00)\end{array}$ & & $95(5.0)$ & \\
\hline \multicolumn{6}{|l|}{ Age (Year) } \\
\hline 10-19 & $3(0.8)$ & $\begin{array}{l}0.91 \\
(0.15)\end{array}$ & $0.176^{\mathrm{a}}$ & $\begin{array}{l}88.33 \\
(16.07)\end{array}$ & $0.006^{\mathrm{a}}$ \\
\hline $20-29$ & $67(18)$ & $\begin{array}{l}0.96 \\
(0.13)\end{array}$ & & $\begin{array}{l}92.91 \\
(9.66)\end{array}$ & \\
\hline $30-39$ & $\begin{array}{l}88 \\
(23.7)\end{array}$ & $\begin{array}{l}0.92 \\
(0.14)\end{array}$ & & $\begin{array}{l}91.59 \\
(11.26)\end{array}$ & \\
\hline $40-49$ & $\begin{array}{l}71 \\
(19.1)\end{array}$ & $\begin{array}{l}0.94 \\
(0.12)\end{array}$ & & $\begin{array}{l}93.45 \\
(8.18)\end{array}$ & \\
\hline $50-59$ & $\begin{array}{l}77 \\
(20.7)\end{array}$ & $\begin{array}{l}0.89 \\
(0.19)\end{array}$ & & $\begin{array}{l}88.38 \\
(11.93)\end{array}$ & \\
\hline$>60$ & $\begin{array}{l}66 \\
(17.7)\end{array}$ & $\begin{array}{l}0.92 \\
(0.15)\end{array}$ & & $\begin{array}{l}87.05 \\
(15.86)\end{array}$ & \\
\hline \multicolumn{6}{|l|}{ Marital status } \\
\hline Married & $\begin{array}{l}307 \\
(82.5)\end{array}$ & $\begin{array}{l}0.92 \\
(0.15)\end{array}$ & $0.165^{\mathrm{b}}$ & $\begin{array}{l}76.31 \\
(10.81)\end{array}$ & $0.405^{\mathrm{b}}$ \\
\hline Not married & $\begin{array}{l}65 \\
(17.5)\end{array}$ & $\begin{array}{l}0.95 \\
(0.11)\end{array}$ & & $\begin{array}{l}76.68 \\
(12.08)\end{array}$ & \\
\hline \multicolumn{6}{|l|}{ Education } \\
\hline Illiterate & $8(2.2)$ & $1.00(0.0)$ & $0.390^{\mathrm{a}}$ & $\begin{array}{l}85.63 \\
(16.35)\end{array}$ & $0.685^{\mathrm{b}}$ \\
\hline Class I to XII & $\begin{array}{l}176 \\
(47.3)\end{array}$ & $\begin{array}{l}0.92 \\
(0.16)\end{array}$ & & $\begin{array}{l}90.08 \\
(11.74)\end{array}$ & \\
\hline Undergraduate & $\begin{array}{l}132 \\
(35.5)\end{array}$ & $\begin{array}{l}0.93 \\
(0.13)\end{array}$ & & $\begin{array}{l}91.17 \\
(09.80)\end{array}$ & \\
\hline Post Graduate & $\begin{array}{l}56 \\
(15.1)\end{array}$ & $\begin{array}{l}0.92 \\
(0.13)\end{array}$ & & $\begin{array}{l}92.75 \\
(07.15)\end{array}$ & \\
\hline \multicolumn{6}{|l|}{ Occupation } \\
\hline Do not work & $22(5.9)$ & $\begin{array}{l}0.91 \\
(0.18)\end{array}$ & $0.205^{\mathrm{a}}$ & $\begin{array}{l}85.23 \\
(19.8)\end{array}$ & $0.518^{\mathrm{a}}$ \\
\hline Domestic work & $\begin{array}{l}103 \\
(27.7)\end{array}$ & $\begin{array}{l}0.89 \\
(0.18)\end{array}$ & & $\begin{array}{l}89.22 \\
(11.9)\end{array}$ & \\
\hline $\begin{array}{l}\text { Clerical, Sales \& } \\
\text { Professional }\end{array}$ & $\begin{array}{l}174 \\
(39.5)\end{array}$ & $\begin{array}{l}0.95 \\
(0.11)\end{array}$ & & $\begin{array}{l}91.98 \\
(10.5)\end{array}$ & \\
\hline Unskilled & $\begin{array}{l}73 \\
(19.6)\end{array}$ & $\begin{array}{l}0.92 \\
(0.17)\end{array}$ & & $\begin{array}{l}91.30 \\
(11.0)\end{array}$ & \\
\hline \multicolumn{6}{|l|}{ Place of Residence } \\
\hline Rural & $\begin{array}{l}156 \\
(41.9)\end{array}$ & $\begin{array}{l}0.93 \\
(0.14)\end{array}$ & $0.929^{\mathrm{a}}$ & $\begin{array}{l}90.74 \\
(12.09)\end{array}$ & $0.352^{\mathrm{a}}$ \\
\hline Urban & $\begin{array}{l}209 \\
(56.2)\end{array}$ & $\begin{array}{l}0.92 \\
(0.16)\end{array}$ & & $\begin{array}{l}90.50 \\
(11.70)\end{array}$ & \\
\hline Do not know & 7 (1.9) & $\begin{array}{l}0.91 \\
(0.15)\end{array}$ & & $\begin{array}{l}95.00 \\
(09.57)\end{array}$ & \\
\hline \multicolumn{6}{|l|}{ BMI } \\
\hline Underweight & $17(4.6)$ & $\begin{array}{l}0.91 \\
(0.18)\end{array}$ & $0.921^{\mathrm{a}}$ & $\begin{array}{l}86.18 \\
(15.66)\end{array}$ & $0.714^{\mathrm{a}}$ \\
\hline Normal & $\begin{array}{l}149 \\
(40.1)\end{array}$ & $\begin{array}{l}0.93 \\
(0.14)\end{array}$ & & $\begin{array}{l}92.8 \\
(09.25)\end{array}$ & \\
\hline Overweight & $\begin{array}{l}147 \\
(39.5)\end{array}$ & $\begin{array}{l}0.92 \\
(\underline{0.16)}\end{array}$ & & $\begin{array}{l}89.73 \\
(14.07)\end{array}$ & \\
\hline Obese & $\begin{array}{l}59 \\
(15.9)\end{array}$ & $\begin{array}{l}\overline{0.95} \\
(\underline{0.10)}\end{array}$ & & $\begin{array}{l}90.59 \\
(09.74)\end{array}$ & \\
\hline \multicolumn{6}{|l|}{ Co-morbidity } \\
\hline Diabetes Mellitus $^{c}$ & $\begin{array}{l}75 \\
(19.4)\end{array}$ & $\begin{array}{l}0.89 \\
(0.19)\end{array}$ & & $\begin{array}{l}87.0 \\
(16.9)\end{array}$ & \\
\hline Hypertension $^{c}$ & $\begin{array}{l}46 \\
(12.4)\end{array}$ & $\begin{array}{l}0.89 \\
(0.19)\end{array}$ & & $\begin{array}{l}85.4 \\
(17.9)\end{array}$ & \\
\hline Heart disease $^{c}$ & $9(2.4)$ & $\begin{array}{l}0.79 \\
(0.30)\end{array}$ & & $\begin{array}{l}82.22 \\
(20.0)\end{array}$ & \\
\hline Kidney disease $^{c}$ & $3(0.8)$ & $\begin{array}{l}0.77 \\
(0.20)\end{array}$ & & $\begin{array}{l}85.0 \\
(08.7)\end{array}$ & \\
\hline Respiratory Disease $^{c}$ & $3(0.8)$ & & & & \\
\hline
\end{tabular}

Table 1 (continued)

\begin{tabular}{|c|c|c|c|c|c|}
\hline \multirow[t]{2}{*}{ Characteristics } & \multirow[t]{2}{*}{ N (\%) } & \multicolumn{2}{|c|}{ EQ-5D-5L Index } & \multicolumn{2}{|c|}{ EQ-5D-5L VAS } \\
\hline & & $\begin{array}{l}\text { Mean } \\
\text { (SD) }\end{array}$ & $\begin{array}{l}\mathrm{P} \\
\text { value }\end{array}$ & Mean (SD) & $\begin{array}{l}\mathrm{P} \\
\text { value }\end{array}$ \\
\hline & & $\begin{array}{l}0.90 \\
(0.17)\end{array}$ & & $\begin{array}{l}83.3 \\
(11.54)\end{array}$ & \\
\hline Cancer $^{c}$ & $1(0.3)$ & $\begin{array}{l}1.00 \\
(1.00)\end{array}$ & & $\begin{array}{l}95.0 \\
(0.00)\end{array}$ & \\
\hline Other illness ${ }^{c}$ & $11(2.9)$ & $\begin{array}{l}0.93 \\
(0.149)\end{array}$ & & $\begin{array}{l}90.69 \\
(11.86)\end{array}$ & \\
\hline
\end{tabular}

SD-Standard Deviation.

$\mathrm{p}$ value from.

a Kruskal Wallis test.

b Mann-Whitney $U$ test.

c Having comorbidity.

pain/discomfort (21.4\%), anxiety/depression (18.4\%), and problem in doing usual activities (17.5\%) than others. Un-married participants reported lower problems in all domains and had higher utility scores than married participants. Participants who were underweight or who had a comorbid illness reported problems in the five dimensions of EQ-5D higher. Overall, men had a higher utility value of $0.938 \pm 0.130$ than women, $0.907 \pm 0.170$. Females reported more problems consistently in all EQ-5D domains against males (Table 2). Gender differences in utility value based on study characteristics were explored, which didn't report any significant difference between groups.

Kruskal-Wallis $\mathrm{H}$ test and Mann-Whitney $U$ test were used to determine if there were significant differences between groups among the characteristics of patients for the EQ-5D-5 L index score and VAS score. The median VAS score was statistically significantly different between age groups in the Kruskal-Wallis $\mathrm{H}$ test, $\mathrm{H}(3)=16.24$, $\mathrm{p}=0.006$. The distribution of the EQ-5D-5L VAS score was similar for all groups, as assessed by visual inspection of boxplots. Subsequently, pairwise comparisons were performed with a Bonferroni correction for multiple comparisons. This post hoc analysis revealed no statistically significant differences in VAS scores between age groups or any other group combination.

EQ-5D-5L index scores for people who reported having CKD and EQ$5 \mathrm{D}$ VAS scores for people who reported having hypertension were statistically significantly higher than those who didn't report the comorbidity, $\mathrm{U}=278, \mathrm{z}=-2.002, \mathrm{p}=0.045$ and $\mathrm{U}=278, \mathrm{z}=-2.313, \mathrm{p}=$ 0.021 in the Mann-Whitney $U$ test. However, distributions of the EQ-5D index scores and EQ-5D VAS scores were not similar among the respective groups, as assessed by visual inspection.

EQ-5D-5L index score and VAS score did not differ between groups in other characteristics of participants reported, such as sociodemographic variables and comorbidities (Table 2).

Based on the requirement of different levels of healthcare the utility values were highest for individuals managed at Covid care centre followed by home-care, private hospital and government hospital. Among those who required hospitalisation the utility values were higher for those who stayed for shorter median duration than the who stayed for long. The details of utility values based on the requirement of healthcare is tabulated in Table 3.

Correlation between the EQ-5D utility and EQ-VAS: We explored the correlation between EQ-5D scores and EQ-VAS scores. We found a moderate, positive, statistically significant correlation between utility and EQ-VAS scores ( rho $=0.574, \mathrm{p}<0.001$ ). The functional level score was directly proportional to both EQ-5D utility scores and EQ-VAS scores. Both scores were increasing while increasing in level function scores of domains and vice versa.

Logistic regression showed that participants in the younger age group (20-29) was associated with a lower likelihood of reporting a problem in the EQ5D domains of mobility [OR $=0.47$ (95\% CI 0.003 to $0.750)$, $\mathrm{p}$-value $=0.031]$ and pain and discomfort $[\mathrm{OR}=0.174(95 \% \mathrm{CI}$ 0.470 to 0.639 ), $\mathrm{p}$-value $=0.008]$. Female participants were associated 
Table 2

Percentage of problems reported in 5 dimensions of EQ-5D.

\begin{tabular}{|c|c|c|c|c|c|c|c|c|c|c|c|c|c|c|c|}
\hline \multirow{2}{*}{$\begin{array}{l}\text { Socio } \\
\text { demographic } \\
\text { Characteristics }\end{array}$} & \multicolumn{3}{|l|}{ Mobility } & \multicolumn{3}{|l|}{$\underline{\text { Self-care }}$} & \multicolumn{3}{|c|}{ Usual Activities } & \multicolumn{3}{|c|}{$\underline{\text { Pain/discomfort }}$} & \multicolumn{3}{|c|}{ Anxiety/depression } \\
\hline & $\begin{array}{l}\text { No Problems } \\
(\%)(\mathrm{n}= \\
350)\end{array}$ & $\begin{array}{l}\text { Problems } \\
(\%)(n=22)\end{array}$ & $\begin{array}{l}\mathrm{P} \\
\text { value }\end{array}$ & $\begin{array}{l}\text { No Problems } \\
(\%)(n= \\
364)\end{array}$ & $\begin{array}{l}\text { Problems } \\
(\%)(n=8)\end{array}$ & $\begin{array}{l}P \\
\text { value }\end{array}$ & $\begin{array}{l}\text { No Problems } \\
(\%)(n= \\
341)\end{array}$ & $\begin{array}{l}\text { Problems } \\
(\%)(n=31)\end{array}$ & $\begin{array}{l}P \\
\text { value }\end{array}$ & $\begin{array}{l}\text { No Problems } \\
(\%)(n= \\
314)\end{array}$ & $\begin{array}{l}\text { Problems } \\
\text { (\%) }(\mathrm{n}=58)\end{array}$ & $\begin{array}{l}P \\
\text { value }\end{array}$ & $\begin{array}{l}\text { No Problems } \\
(\%)(\mathrm{n}= \\
326)\end{array}$ & $\begin{array}{l}\text { Problems } \\
(\%)(n=46)\end{array}$ & $\begin{array}{l}P \\
\text { value }\end{array}$ \\
\hline \multicolumn{16}{|l|}{ Age (Years) } \\
\hline $10-19$ & 66.7 & 33.3 & 0.115 & 100 & 0.0 & 0.330 & 100 & 0.0 & 0.745 & 100 & 0.0 & 0.012 & 100 & 0.0 & 0.252 \\
\hline $20-29$ & 95.5 & 4.5 & & 95.5 & 4.5 & & 95.5 & 4.5 & & 95.5 & 4.5 & & 92.5 & 7.5 & \\
\hline $30-39$ & 97.7 & 2.3 & & 98.9 & 1.1 & & 89.8 & 10.2 & & 79.5 & 20.5 & & 89.8 & 10.2 & \\
\hline $40-49$ & 94.4 & 5.6 & & 100 & 0.0 & & 93.0 & 7.0 & & 90.1 & 9.9 & & 88.7 & 11.3 & \\
\hline 50-59 & 89.6 & 10.4 & & 96.1 & 3.9 & & 90.9 & 9.1 & & 79.2 & 20.8 & & 79.2 & 20.8 & \\
\hline$>60$ & 93.9 & 6.1 & & 98.5 & 1.5 & & 89.4 & 10.6 & & 78.8 & 21.2 & & 87.9 & 12.1 & \\
\hline \multicolumn{16}{|l|}{ Gender } \\
\hline Male & 94.9 & 5.1 & 0.589 & 98.6 & 1.4 & 0.333 & 94.9 & 5.1 & 0.36 & 86.9 & 13.1 & 0.195 & 89.7 & 10.3 & 0.264 \\
\hline Female & 92.9 & 7.1 & & 96.8 & 3.2 & & 87.1 & 12.9 & & 80.6 & 19.4 & & 84.5 & 15.5 & \\
\hline Other & 100 & 0.0 & & 100 & 0.0 & & 100 & 0.0 & & 100 & 0.0 & & 100 & 0.0 & \\
\hline \multicolumn{16}{|l|}{ Marital status } \\
\hline Married & 93.5 & 6.5 & 0.393 & 97.7 & 2.3 & 1.000 & 90.9 & 9.1 & 0.324 & 83.1 & 16.9 & 0.135 & 86.0 & 14.0 & 0.38 \\
\hline Not married & 96.9 & 3.1 & & 98.5 & 1.5 & & 95.4 & 4.6 & & 90.8 & 9.2 & & 95.4 & 4.6 & \\
\hline \multicolumn{16}{|l|}{ Education } \\
\hline Illiterate & 100 & 0.0 & 0.125 & 100 & 0.0 & 0.756 & 100 & 0.0 & 0.984 & 100 & 0.0 & 0.619 & 100 & 0.0 & 0.019 \\
\hline Class I to XII & 92.0 & 8.0 & & 98.3 & 1.7 & & 91.5 & 8.5 & & 82.4 & 17.6 & & 86.4 & 13.6 & \\
\hline Undergraduate & 93.9 & 6.1 & & 97.7 & 2.3 & & 90.9 & 9.1 & & 84.8 & 15.2 & & 84.1 & 15.9 & \\
\hline Post Graduate & 100 & 0.0 & & 96.4 & 3.6 & & 92.9 & 7.1 & & 87.5 & 12.5 & & 98.2 & 1.8 & \\
\hline \multicolumn{16}{|l|}{ Occupation } \\
\hline Do not work & 90.9 & 9.1 & 0.017 & 100 & 0.0 & 0.083 & 95.5 & 4.5 & 0.003 & 86.4 & 13.6 & 0.228 & 86.4 & 13.6 & 0.168 \\
\hline Domestic work & 92.2 & 7.8 & & 95.1 & 4.9 & & 82.5 & 17.5 & & 78.6 & 21.4 & & 81.6 & 18.4 & \\
\hline $\begin{array}{l}\text { Clerical, Sales \& } \\
\text { Professional }\end{array}$ & 97.7 & 2.3 & & 99.4 & 0.6 & & 94.8 & 5.2 & & 87.9 & 12.1 & & 90.2 & 9.8 & \\
\hline Unskilled & 89.0 & 11.0 & & 97.3 & 2.3 & & 95.9 & 4.1 & & 83.6 & 16.4 & & 90.4 & 9.6 & \\
\hline \multicolumn{16}{|l|}{$\begin{array}{l}\text { Place of } \\
\text { Residence }\end{array}$} \\
\hline Rural & 92.9 & 7.1 & 0.247 & 98.1 & 1.9 & 1.000 & 94.9 & 5.1 & 0.104 & 84.6 & 15.4 & 1.000 & 84.3 & 15.4 & 1.000 \\
\hline Urban & 95.2 & 4.8 & & 97.6 & 2.4 & & 89.5 & 10.5 & & 84.2 & 15.8 & & 84.2 & 15.8 & \\
\hline Do not know & 85.7 & 14.3 & & 100 & 0.0 & & 85.7 & 14.3 & & 85.7 & 14.3 & & 85.7 & 14.3 & \\
\hline \multicolumn{16}{|l|}{ BMI } \\
\hline Underweight & 82.4 & 17.6 & 0.112 & 100 & 0.0 & 1.000 & 82.4 & 17.6 & 0.382 & 76.5 & 23.5 & 0.308 & 82.4 & 17.6 & 0.735 \\
\hline Normal & 92.6 & 7.4 & & 97.3 & 2.7 & & 91.9 & 8.1 & & 83.9 & 16.1 & & 89.3 & 10.7 & \\
\hline Overweight & 95.9 & 4.1 & & 98.0 & 2.0 & & 91.2 & 8.8 & & 83.0 & 17.0 & & 87.1 & 12.9 & \\
\hline Obese & 96.6 & 3.4 & & 98.3 & 1.7 & & 94.9 & 5.1 & & 91.5 & 8.5 & & 86.4 & 13.6 & \\
\hline \multicolumn{16}{|l|}{ Co-Morbid Illness } \\
\hline Diabetes Mellitus & 92.0 & 8.0 & 0.393 & 96.0 & 4.0 & 0.218 & 86.7 & 13.3 & 0.100 & 76.0 & 24.0 & 0.032 & 82.7 & 17.3 & 0.168 \\
\hline Hypertension & 93.5 & 6.5 & 0.744 & 95.7 & 4.3 & 0.259 & 87.0 & 13.0 & 0.249 & 76.1 & 23.9 & 0.126 & 80.4 & 19.6 & 0.147 \\
\hline Heart disease & 88.9 & 11.1 & 0.426 & 88.9 & 11.1 & 0.179 & 66.7 & 33.3 & 0.031 & 66.7 & 33.3 & 0.151 & 66.7 & 33.3 & 0.087 \\
\hline Kidney disease & 66.7 & 33.3 & 0.168 & 100 & 0.0 & 1.000 & 66.7 & 33.3 & 0.230 & 33.3 & 66.7 & 0.065 & 66.7 & 33.3 & 0.328 \\
\hline $\begin{array}{l}\text { Respiratory } \\
\text { Disease }\end{array}$ & 100.0 & 0.0 & 1.000 & 100 & 0.0 & 1.000 & 66.7 & 33.3 & 0.230 & 66.7 & 33.3 & 0.400 & 100 & 0.0 & 1.000 \\
\hline Cancer & 100.0 & 0.0 & 1.000 & 100 & 0.0 & 1.000 & 100 & 0.0 & 1.000 & 100 & 0.0 & 1.000 & 100 & 0.0 & 1.000 \\
\hline Other illness & 47.6 & 52.4 & 0.493 & 100 & 0.0 & 1.000 & 100 & 0.0 & 0.610 & 81.8 & 18.2 & 0.684 & 90.9 & 9.1 & 1.000 \\
\hline
\end{tabular}

$\mathrm{P}$ values are the probability of Fischer's exact test. Bold values were statistically significant at 0.05 . 
Table 3

Characteristics of respondents and EQ-5D index and visual analogue (VAS) scores based on requirement of healthcare.

\begin{tabular}{|c|c|c|c|}
\hline & $\mathrm{n}(\%)$ & $\begin{array}{l}\text { Utility score } \\
(\text { Mean } \pm \text { SD) }\end{array}$ & $\begin{array}{l}\text { EQ-VAS (Mean } \\
\pm \mathrm{SD})\end{array}$ \\
\hline \multicolumn{4}{|c|}{ Place of treatment } \\
\hline \multicolumn{4}{|c|}{ Home } \\
\hline Yes & $\begin{array}{l}309 \\
(83.1)\end{array}$ & $0.929 \pm 0.14$ & $91.29 \pm 0$ \\
\hline No & $\begin{array}{l}63 \\
(16.9)\end{array}$ & $0.907 \pm 0.19$ & $87.70 \pm 0$ \\
\hline \multicolumn{4}{|c|}{ COVID Care Centre } \\
\hline Yes & $22(5.9)$ & $0.934 \pm 0.13$ & $92.05 \pm 9.7$ \\
\hline No & $\begin{array}{l}350 \\
(94.1)\end{array}$ & $0.925 \pm 0.15$ & $90.60 \pm 0$ \\
\hline \multicolumn{4}{|c|}{$\begin{array}{l}\text { Days of stay in COVID Care } \\
\text { Centre }\end{array}$} \\
\hline Below Median & $13(59.0)$ & $0.941 \pm 0.15$ & $93.08 \pm 12.0$ \\
\hline Above Median & $9(40.9)$ & $0.925 \pm 0.12$ & $90.56 \pm 05.3$ \\
\hline \multicolumn{4}{|l|}{ Govt Hospital } \\
\hline Yes & $\begin{array}{l}154 \\
(41.4)\end{array}$ & $0.897 \pm 0.17$ & $88.77 \pm 12.7$ \\
\hline No & $\begin{array}{l}218 \\
(58.6)\end{array}$ & $0.946 \pm 0.13$ & $92.04 \pm 0$ \\
\hline \multicolumn{4}{|c|}{ Days of stay in Govt Hospital } \\
\hline Below Median & $\begin{array}{l}88 \\
(57.1)\end{array}$ & $0.9184 \pm 0.14$ & $89.32 \pm 14.4$ \\
\hline $\begin{array}{l}\text { Above Median } \\
\text { Private Hospi }\end{array}$ & $66(42.9)$ & $0.8676 \pm 0.12$ & $88.03 \pm 10.1$ \\
\hline Yes & $\begin{array}{l}117 \\
(31.5)\end{array}$ & $0.923 \pm 0.16$ & $90.64 \pm 0$ \\
\hline No & $\begin{array}{l}255 \\
(68.5)\end{array}$ & $0.927 \pm 0.15$ & $90.71 \pm 0$ \\
\hline \multicolumn{4}{|c|}{ Days of stay in private } \\
\hline Below Median & $81(69.2)$ & $0.930 \pm 0.14$ & $92.28 \pm 11.1$ \\
\hline Above Median & $36(30.8)$ & $0.907 \pm 0.19$ & $86.94 \pm 17.9$ \\
\hline \multicolumn{4}{|c|}{ Requirement of Additional care } \\
\hline \multicolumn{4}{|c|}{ ICU-care } \\
\hline Yes & $14(3.8)$ & $0.808 \pm 0.24$ & $86.79 \pm 0$ \\
\hline No & $\begin{array}{l}358 \\
(96.2)\end{array}$ & $0.940 \pm 0.13$ & $91.58 \pm 0$ \\
\hline \multicolumn{4}{|c|}{ Mechanical ventilation } \\
\hline Yes & $11(2.9)$ & $0.814 \pm 0.27$ & $86.36 \pm 1$ \\
\hline No & $\begin{array}{l}361 \\
(97.1)\end{array}$ & $0.939 \pm 0.13$ & $91.57 \pm 0$ \\
\hline \multicolumn{4}{|c|}{ Oxygen Supplementation } \\
\hline Yes & $14(3.8)$ & $0.723 \pm 0.23$ & $82.86 \pm 0$ \\
\hline No & $\begin{array}{l}358 \\
(96.2)\end{array}$ & $0.942 \pm 0.13$ & $91.69 \pm 0$ \\
\hline
\end{tabular}

with an increased likelihood of reporting a problem in the EQ5D domain of usual activities [OR $=2.734$ (95\% CI 1.269 to 5.889), p-value 0.010] against males. All other variables were not associated with any EQ-5D domains.

Multivariate regression analysis: We dichotomized each dimension of EQ-5D, used as the dependent variable in the multivariate logistic regression analysis. Gender, age, marital status, education, occupation, residence, and BMI were included as independent variables; multivariate logistic regression models were conducted. Only those variables that exerted a significant relationship with any dimension from EQ-5D were reported in Supplementary Table 2. EQ-5D index score was significantly influenced by occupation [OR $=1.119(95 \%$ CI 1.018 to 1.391$)$ ], gender [OR $=2.179(95 \% \mathrm{CI} 1.007$ to 4.714$)]$ and place of residence [OR = 2.146 (95\% CI 1.023 to 4.503$)$ ].

\section{Discussion}

This is the first study that assessed the utility value by EQ-5D -5L among COVID-19 recovered individuals in an Indian context. In our study utility score was lower (0.93) than the general population. This study corroborates with the evidence from the recent EQ-5D studies from China, ${ }^{8}$ the USA, ${ }^{15}$ Iran, ${ }^{16}$ and Switzerland, ${ }^{17}$ which reported lower utility value in comparison with the general population. An interesting finding is that men had higher utility scores than women. Further, the gender difference in the severity of illness broadly supports the other studies in this area linking with gender. ${ }^{18,19}$ Like previous studies, our study also corroborates with the idea that there is an observed difference in the quality of life between men and women. ${ }^{20}$ In our study, the strength of the correlations of the utility value and EQ-VAS was moderately stronger.

Another prominent finding which supports previous similar EQ5D studies is that this study also found that the Health-related Quality of life is associated with Comorbid conditions. The presence of comorbidities is associated with a poor outcome in patients with COVID-19. Our study found that participants with comorbidities reported lower EQ5D scores. Diabetes prevalence is highest in Indian COVID-19 patients ${ }^{21}$ compared to other countries, and our study findings suggest the same. Our study findings were similar to those of Japanese individuals suffering from diabetes mellitus with a reported utility score of $0.846^{22}$ and another study that reported a utility score of cardiovascular diseases as $0.84 .^{23}$

Interestingly, in all domains of EQ-5D, the majority of the participants reported a lesser number of problems with mobility, self-care, and usual activities. Our study also revealed that few patients had severe pain/discomfort and suffered from severe anxiety/depression similar to individuals affected with MERS ${ }^{24}$ and SARS ${ }^{25,}{ }^{22}$ Our study supports the findings of the study that women were more anxious than men regarding COVID-19, ${ }^{26}$ and another study ${ }^{27}$ found that by reducing anxiety and depression in individuals, there is a notable rise in the QoL. Overall, no significant association was found between demographic characteristics and quality of life, other than a significant association in occupation groups concerning utility value.

Limitation: Our study had few limitations. When our test of normality showed that the distribution of data is non-normal and positively skewed for both EQ-5D-5L Utility score and EQ-VAS, it was customary to use the median instead of the mean as the greater emphasis should be placed on using the median as opposed to the mean. However, we reported the mean since the difference between the median and mean were not appreciably different (a subjective assessment), and it allows easier comparisons with previous research works. Self-reported patient outcomes can be subjected to an individual's perception, beliefs, culture, socioeconomic status, Health care system, and social structure. Self-reports can be less accurate, and participants can not accurately depict how they genuinely feel about their status, leading to information Bias. The majority of the study participants were male and residing in urban areas. This may limit the generalizability of the study findings. If the information on the socioeconomic status in terms of gross income and Out of pocket expenditure for treatment have been collected from our study participants, it would have provided an added value to our research.

Conclusion: This study focussed on assessing the Health-related quality of life in COVID-19 individuals by the EQ-5D-5L tool. This study showed that there was a significant reduction in utility scores than the general population. A significant percentage of COVID-19 recovered individuals reported pain/discomfort and anxiety/depression; hence, effective awareness programs and interventions are needed to increase their quality of life. The association of socio-demographic characteristics with Health-related quality of life further imparts the need for a patientcentered approach to delivering health care and disease management programs.

\section{Ethics approval and consent to participate}

Yes, Approval was taken from ICMR-National Institute of Epidemiology Institutional Human Ethics committee (NIE/IHEC/202010-02), and informed consent was taken from all the study participants. 


\section{Consent for publication}

All authors have reviewed and consented for publication.

\section{Availability of data and materials}

All the group level data is included in the publication.

\section{Conflicts of interest}

None.

\section{Funding}

We received no specific funding for this work. However, the Dept. of Health Research Govt. of India funds the Health Technology Assessment resource centre ICMR-NIE. Funders had no role in the conceptualisation, conduction, and manuscript preparation.

\section{Authors contribution}

Barani S: Conceptualisation, Data curation, Formal analysis, Methods, Original draft.

Bhatnagar T: Conceptualisation, Data curation, Methods, Review \& editing.

Natarajan M: Data curation, Formal analysis, Methods, Review \& editing.

Kumari G: Data curation, Review \& editing.

Sonekar HB: Data curation, Review \& editing.

Sasidharan A: Analysis, Review \& editing.

TS Selvavinayagam: Critical Review \& editing.

Bagepally BS: Conceptualisation, Formal analysis, Methods, Review \& editing.

\section{Acknowledgements}

The authors acknowledge the following from ICMR-National Institute of Epidemiology - P. Lourdu Stella Mary, I. Kalaimani, R. Harikrishnan, T. Ravichandran, R.Gopinath, D.Murugan, K.Sathish Kumar, K. Ramu, V.Ramesh, M.Anthony Doss, J.Amudhini, and S.K.Kanthimathi for telephone-based electronic data collection; R. Sabarinathan, D. Chokkalingam and A. Suresh for technical support; for coordinating the data collection.

\section{Appendix A. Supplementary data}

Supplementary data to this article can be found online at https://doi. org/10.1016/j.cegh.2021.100943.

\section{References}

1 Zhu N, Zhang D, Wang W, et al. A novel coronavirus from patients with pneumonia in China, 2019. N Engl J Med. 2020;382(8):727-733. https://doi.org/10.1056/ NEJMoa2001017.

2 WHO Coronavirus (COVID-19) Dashboard. Accessed June 13, 2021. https://covid19. who.int.

3 Covid-19 Tamil-nadu update (LIVE) : 2353721 cases and 29547 deaths from coronavirus outbreak in India - corona clusters | historical data \& daily charts of corona virus in Indian states. TrulyMadly. Accessed June 13, 2021.//coronaclusters. in/tamil-nadu.
4 Azizi A, Achak D, Aboudi K, et al. Health-related quality of life and behavior-related lifestyle changes due to the COVID-19 home confinement: dataset from a Moroccan sample. Data Brief. 2020;32, 106239. https://doi.org/10.1016/j.dib.2020.106239.

5 Qu G, Zhen Q, Wang W, et al. Health-related quality of life of COVID-19 patients after discharge: a multicenter follow-up study. J Clin Nurs. 2021;30(11-12):1742-1750. https://doi.org/10.1111/jocn.15733.

6 Kharshiing KD, Kashyap D, Gupta K, et al. Quality of life in the COVID-19 pandemic in India: exploring the role of individual and group variables. Community Ment Health J. 2021;57(1):70-78, https://doi.org/10.1007/s10597-020-00712-6.

7 Devlin NJ, Shah KK, Feng Y, Mulhern B, van Hout B. Valuing health-related quality of life: an EQ-5D-5L value set for England. Health Econ. 2018;27(1):7-22. https://doi. org $/ 10.1002 / \mathrm{hec} .3564$.

8 Ping W, Zheng J, Niu X, et al. Evaluation of health-related quality of life using EQ-5D in China during the COVID-19 pandemic. PLoS One. 2020;15(6), e0234850. https:// doi.org/10.1371/journal.pone.0234850.

9 von Elm E, Altman DG, Egger M, Pocock SJ, Gøtzsche PC, Vandenbroucke JP. The Strengthening the Reporting of Observational Studies in Epidemiology (STROBE) statement: guidelines for reporting observational studies. J Clin Epidemiol. 2008;61 (4):344-349. https://doi.org/10.1016/j.jclinepi.2007.11.008.

10 Gandhi M, Xu Y, Luo N, Cheung YB. Sample size determination for EQ-5D-5L value set studies. Qual Life Res. 2017;26(12):3365-3376. https://doi.org/10.1007/s11136017-1685-3.

11 Whitehead SJ, Ali S. Health outcomes in economic evaluation: the QALY and utilities. Br Med Bull. 2010;96(1):5-21. https://doi.org/10.1093/bmb/ldq033.

12 Emrani Z, Akbari Sari A, Zeraati H, Olyaeemanesh A, Daroudi R. Health-related quality of life measured using the EQ-5D-5 L: population norms for the capital of Iran. Health Qual Life Outcome. 2020;18(1):108. https://doi.org/10.1186/s12955020-01365-5.

13 Yin S, Njai R, Barker L, Siegel PZ, Liao Y. Summarizing health-related quality of life (HRQOL): development and testing of a one-factor model. Popul Health Metrics. 2016; 14:22. https://doi.org/10.1186/s12963-016-0091-3.

14 van Hout B, Janssen MF, Feng Y-S, et al. Interim scoring for the EQ-5D-5L: mapping the EQ-5D-5L to EQ-5D-3L value sets. Value Health. 2012;15(5):708-715. https:// doi.org/10.1016/j.jval.2012.02.008.

15 Hay JW, Gong CL, Jiao X, et al. A US population health survey on the impact of COVID-19 using the EQ-5D-5L. J Gen Intern Med. 2021;36(5):1292-1301. https:// doi.org/10.1007/s11606-021-06674-Z.

16 Arab-Zozani M, Hashemi F, Safari H, Yousefi M, Ameri H. Health-related quality of life and its associated factors in COVID-19 patients. Osong Public Health Res Perspect. 2020;11(5):296-302. https://doi.org/10.24171/j.phrp.2020.11.5.05.

17 Poteet S, Craig BM. QALYs for COVID-19: a comparison of US EQ-5D-5L value sets. Patient. 2021;14(3):339-345. https://doi.org/10.1007/s40271-021-00509-z.

$18 \mathrm{Vu}$ MQ, Tran TTP, Hoang TA, Khuong LQ, Hoang MV. Health-related quality of life of the Vietnamese during the COVID-19 pandemic. PLoS One. 2020;15(12), e0244170. https://doi.org/10.1371/journal.pone.0244170.

19 Jin JM, Bai P, He W, et al. Gender differences in patients with COVID-19: focus on severity and mortality. Front Public Health. 2020;8(152):152. https://doi.org/ 10.3389/fpubh.2020.00152.

20 Gebhard C, Regitz-Zagrosek V, Neuhauser HK, Morgan R, Klein SL. Impact of sex and gender on COVID-19 outcomes in Europe. Biol Sex Differ. 2020;11(1):29. https://doi. org/10.1186/s13293-020-00304-9.

21 Singh AK, Misra A. Impact of COVID-19 and comorbidities on health and economics: focus on developing countries and India. Diabetes, Metab Syndrome : Clin Res Rev. 2020;14(6):1625-1630. https://doi.org/10.1016/j.dsx.2020.08.032.

22 Sakamaki H, Ikeda S, Ikegami N, et al. Measurement of HRQL using EQ-5D in patients with type 2 diabetes mellitus in Japan. Value Health. 2006;9(1):47-53. https://doi.org/10.1111/j.1524-4733.2006.00080.x.

23 Tran BX, Moir MP, Thai TPT, et al. Socioeconomic inequalities in health-related quality of life among patients with cardiovascular diseases in vietnam. BioMed Res Int. 2018, 2643814. https://doi.org/10.1155/2018/2643814, 2018.

24 Ahmed H, Patel K, Greenwood DC, et al. Long-term clinical outcomes in survivors of severe acute respiratory syndrome and Middle East respiratory syndrome coronavirus outbreaks after hospitalisation or ICU admission: a systematic review and meta-analysis. J Rehabil Med. 2020;52(5), jrm00063. https://doi.org/10.2340/ 16501977-2694.

25 Lam MH, Wing YK, Yu MW, et al. Mental morbidities and chronic fatigue in severe acute respiratory syndrome survivors: long-term follow-up. Arch Intern Med. 2009; 169(22):2142-2147. https://doi.org/10.1001/archinternmed.2009.384.

26 Greenhawt M, Kimball S, DunnGalvin A, et al. Media influence on anxiety, health utility, and health beliefs early in the SARS-CoV-2 pandemic-a survey study. $J$ Gen Intern Med. 2021;36(5):1327-1337. https://doi.org/10.1007/s11606-020-06554-y.

27 Bodrova RA, Kuchumova TV, Zakamyrdina AD, Yunusova ER, Fadeev GY. Efficacy of low-frequency magnetic therapy in patients with COVID-19 pneumonia. Vopr Kurortol Fizioter Lech Fiz Kul't. 2020;97(6):11-16. https://doi.org/10.17116/ kurort20209706111. 